\title{
Use of magnetic resonance imaging for evaluation of therapeutic response in breast cancer-related lymphedema: A systematic review
}

\author{
Antonio Jorge Forte ${ }^{1}$, Daniel Boczar ${ }^{1}$, Salam Kassis ${ }^{2}$, Maria T. Huayllani ${ }^{1}$, Sarah A. McLaughlin ${ }^{3}$ \\ ${ }^{1}$ Division of Plastic Surgery, Mayo Clinic, Jacksonville, FL; ${ }^{2}$ Department of Plastic Surgery, Vanderbilt University Medical Center, Nashville, \\ TN; ${ }^{3}$ Department of Surgery, Mayo Clinic, Jacksonville, FL, USA
}

Breast cancer treatment-related lymphedema (BCRL) is a common comorbidity in breast cancer survivors. Although magnetic resonance imaging (MRI) is widely used to evaluate therapeutic response of patients with various medical conditions, it is not routinely used to evaluate lymphedema patients. We conducted a systematic review of the literature to identify studies on the use of MRI to evaluate therapy for BCRL. We hypothesized that MRI could provide information otherwise not possible through other examinations. On October 21, 2019, we conducted a systematic review on the PubMed/MEDLINE and Scopus databases, without time frame or language limitations, to identify studies on the use of MRI to evaluate therapy for BCRL. We excluded studies that investigated other applications of MRI, such as lymphedema diagnosis and surgical planning. Of 63 potential articles identified with the search, three case series fulfilled the eligibility criteria. In total, 53 patients with BCRL were included and quantitatively evaluated with MRI before and after manual lymphatic drainage. Authors used MRI or MR lymphagiography to investigate factors such as lymphatic vessel cross-sectional area, tissue water relaxation time $\left(\mathrm{T}_{2}\right)$, and chemical exchange saturation transfer. The only study that compared MRI measurement with standard examinations reported that MRI added information to the therapy evaluation. MRI seems to be a promising tool for quantitative measurement of therapeutic response in patients with BCRL. However, the identified studies focused on only manual lymphatic drainage and were limited by the small numbers of patients. More studies are necessary to shed light on the topic.

Keywords Breast cancer lymphedema / Lymphedema / Magnetic resonance imaging
Correspondence: Antonio Jorge Forte Division of Plastic Surgery, Mayo Clinic, 4500 San Pablo Rd, Jacksonville, FL 32224, USA Tel: +1-904-953-2073 Fax: +1-904-953-6489 E-mail: ajvforte@yahoo.com.br

This study was supported in part by the Mayo Clinic Center for Individualized Medicine, and the Plastic Surgery Foundation.

\section{INTRODUCTION}

Secondary lymphedema is a life-long condition promoted by lymphatic injury after cancer treatment, such as radiation and axillary lymph node dissection [1-5]. Breast cancer treatmentrelated lymphedema (BCRL) is a common comorbidity in breast cancer survivors that could affect up to $65 \%$ of patients [5-7].

Early detection and management are key elements to minimizing lymphedema progression, but relevant biomarkers of lymphedema remain unknown [8]. Imaging biomarkers that identify fibrosis may also identify patients who need more-ag- 
gressive therapies [9]. However, few methods for quantifying lymphatic function have been proposed [10]. For example, volumetric measurements, which are often used in clinical practice, are mainly capable of differentiating affected from nonaffected limbs $[8,11]$.

Although magnetic resonance imaging (MRI) is widely used to evaluate therapeutic response of patients with various medical conditions, the examination is not routinely used to evaluate lymphedema patients [8]. With the evolution of MRI technology, it can support the development of methods for recording functional and structural changes in lymphedema tissue [8]. Therefore, we performed a systematic review of the literature on the use of MRI for therapy evaluation in BCRL. We hypothesized that MRI could provide relevant information otherwise not possible through other examinations.

\section{SEARCH STRATEGY}

We followed the guidelines outlined in the Preferred Reporting Items for Systematic Reviews and Meta-Analyses (PRISMA). On October 21, 2019, two authors (DB and MTH) independently searched the PubMed/MEDLINE and Scopus databases, without time frame or language limitations, for the following Medical Subject Headings (MeSH) terms: ("magnetic resonance imaging" OR "MRI”) AND "breast cancer lymphedema." The title and abstract of each identified article were initially screened, and then the full text was reviewed. The reference lists of the studies that fulfilled the study eligibility criteria (see the Selection Criteria section below) were also examined to identify articles not identified with our initial search. Duplicate articles were excluded, and disagreements regarding article identification and inclusion were resolved by another author (AJF).

\section{SELECTION CRITERIA}

We included studies that met eligibility criteria and reported data about the use of MRI to evaluate therapy for BCRL. Therefore, we excluded studies that investigated other applications of $\mathrm{MRI}$, such as lymphedema diagnosis and surgical planning. Abstracts, presentations, reviews, and meta-analyses were also excluded.

\section{DATAEXTRACTIONANID PROCESSING}

We extracted data about year of the publication, country, study design, level of evidence, study population, therapy, type of MRI, measurements, and comparative examinations. Two authors (DB and MTH) extracted data from the text, tables, and figures of each article, and another author (AJF) confirmed the accuracy of data entry.

\section{STUDY CHARACTERISTICS}

Of 63 potential articles identified with our search, three case series performed in the United States fulfilled the eligibility criteria (Table 1, Fig. 1) $[8,12,13]$. In total, 53 patients with BCRL were included and quantitatively evaluated with 3.0-T MRI before and after manual lymphatic drainage (MLD). Only one study compared MRI findings with those of other standard examinations [8]. Three different techniques were proposed, and all studies showed the feasibility of using MRI to quantitatively measure lymphedema therapy responses.

\section{MAGNETIC RESONANCE LIMPHANGIOGRAPHIY}

In 2017, Crescenzi et al. [12] proposed a 3.0-T magnetic resonance lymphangiography sequence without exogenous contrast agents to detect lymph stasis. They used a long 3.0-T relaxation time and a low flow velocity of lymphatic fluid. They enrolled 25 patients with unilateral BCRL, and a subgroup of fine patients underwent MLD. Maximal lymphatic vessel cross-sec-

Table 1. Summary of the studies

\begin{tabular}{|c|c|c|c|c|c|c|c|c|c|}
\hline Author & Year & Country & Study type & $\begin{array}{l}\text { Level of } \\
\text { evidence }\end{array}$ & Patient & Therapy & Method & Measure & Comparison exam \\
\hline Crescenzi et al. [12] & 2017 & USA & Case series & Level III & $\begin{array}{l}25 \text { Patients with BCRL; } \\
\text { subgroup of } 5 \text { patients } \\
\text { underwent MLD }\end{array}$ & MLD & MRL & $\begin{array}{l}\text { Lymphatic } \\
\text { vessel cross- } \\
\text { sectional area }\end{array}$ & None \\
\hline Donahue et al. [8] & 2017 & USA & Case series & Level II & 16 Patients with BCRL & MLD & MRI & $\begin{array}{l}\text { Tissue water } \\
\text { relaxation time } \\
\left(\mathrm{T}_{2}\right)\end{array}$ & $\begin{array}{l}\text { Non-MRI measures: limb } \\
\text { volume, tissue dielectric } \\
\text { constant, and bioimpedance }\end{array}$ \\
\hline Crescenzi et al. [13] & 2019 & USA & Case series & Level III & 12 Patients with BCRL & MLD & MRI & CEST & None \\
\hline
\end{tabular}

BCRL, breast cancer treatment-related lymphedema; MLD, manual lymphatic drainage; MRL, magnetic resonance lymphangiography; MRI, magnetic resonance imaging; CEST, chemical exchange saturation transfer. 


\section{Fig. 1. PRISMA flow diagram}

PRISMA, Preferred Reporting Items for Systematic Reviews and Meta-Analyses.
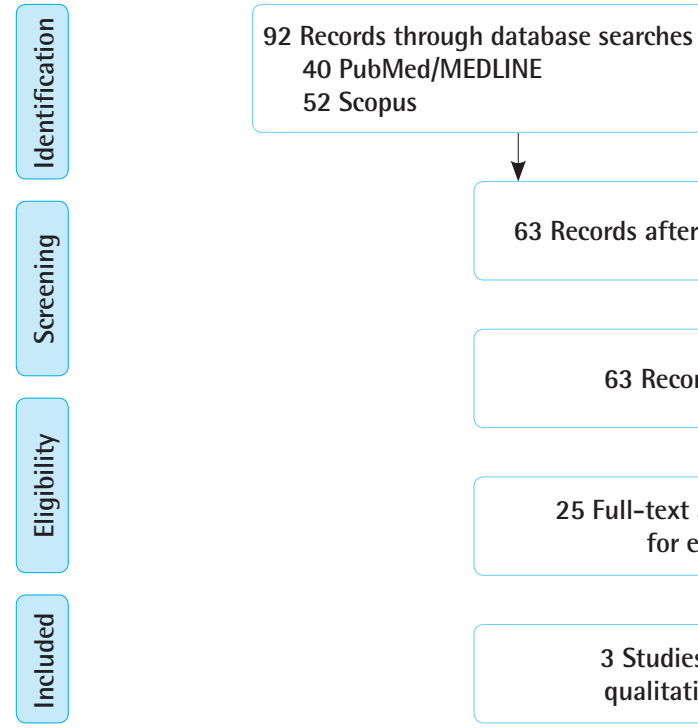

0 Additional records identified through other sources tional area was recorded before and after MLD. Difference of lymphatic cross-sectional areas between affected and nonaffected arms and torso were statistically significant among patient volunteers (arm: $\mathrm{P}=0.010$; torso: $\mathrm{P}=0.025$ ). Moreover, in the subgroup who underwent MLD, it was possible to detect a reduction in lymphatic vessel cross-sectional area in the torso after therapy (mean \pm standard deviation [SD]: pre-MLD, $31.4 \pm 26.0$ $\mathrm{mm}^{2}$; post-MLD, $23.1 \pm 24.3 \mathrm{~mm}^{2} ; \mathrm{P}=0.036$ ). Their study was limited because of the small group of patients and no comparison with a standard examination, such as fluoroscopy. Moreover, the examined spatial resolution did not identify lymphatic vessels with cross-sectional area less than $2 \mathrm{~mm}^{2}$, so this method was sensitive mainly for evaluation of lymphedema patients with engorged lymphatic vessels [12].

\section{MAGNETIC RESONANCE IMAGING}

In 2017, Donahue et al. [8] proposed using MRI to measure tissue water relaxation time $\left(\mathrm{T}_{2}\right)$ and identify tissue changes after MLD. Sixteen patients with BCRL were enrolled. Non-MRI measures (limb volume, tissue dielectric constant, and bioimpedance) were compared with MRI measures (qualitative 3.0-T diffusion-weighted, $\mathrm{T}_{1}$-weighted and $\mathrm{T}_{2}$-weighted $\mathrm{MRI}$ and quantitative multi-echo $\mathrm{T}_{2} \mathrm{MRI}$ of the axilla) before and after MLD. Non-MRI measures could capture differences between affected and nonaffected arms but not the change in response to MLD. However, deep-tissue $\mathrm{T}_{2}$-weighted MRI captured a change after
MLD: in affected arms, mean \pm SD before, $0.0371 \pm 0.003$ seconds; after, $0.0389 \pm 0.003$ seconds $(\mathrm{P}=0.029)$; in nonaffected arms, mean \pm SD before, $0.0365 \pm 0.002$ seconds; after, $0.0395 \pm$ 0.002 seconds $(\mathrm{P}<0.01)$. The authors also noted that patients with stage two lymphedema had a higher $\mathrm{T}_{2}$ increase in their affected arms after MLD than patients with stages zero and one lymphedema. However, this study was limited by the small number of patients and because MRI was performed immediately after MLD, and therefore, only immediate changes promoted by MLD were assessed [8].

In 2019, Crescenzi et al. [13] proposed 3.0-T MRI to quantify the effect of MLD by differences of chemical exchange saturation transfer (CEST) contrast, using correction procedures for $\mathrm{B}_{0}$ and $B_{1}$ heterogeneity and $T_{1}$ relaxation time. Twelve patients with BCRL were enrolled and evaluated with MRI before and after MLD. They bilaterally calculated, in the axilla, $B_{1}$ efficiency and $\mathrm{T}_{1}$ efficiency with CEST (proton transfer ratio [PTR'], magnetization transfer ratio asymmetry, and apparent exchange-dependent relaxation). PTR' significantly increased in the affected arm, after MLD (before mean \pm SD, $0.41 \pm 0.05$ vs. after mean $\pm S D$, $0.43 \pm 0.03 ; \mathrm{P}=0.02)$. This finding was interpreted as a consequence of mobilized lymphatic fluid. This study was limited by the small, heterogeneous group of patients with BCRL [13].

\section{DISCUSSION}

In the present systematic review, we noted that the scientific lit- 
erature about the use of MRI for therapy evaluation in BCRL can be summarized in three case series from the United States. These studies involved a total of 53 patients and used 3.0-T MRI to evaluate the effect of MLD therapy using 3.0-T MRI. Although all studies proposed quantitative measurements, different techniques were used to measure lymphatic vessel crosssectional area, $\mathrm{T}_{2}$, and CEST. The three studies reached their goal to investigate the feasibility of quantitative MRI for lymphedema therapy evaluation. In only one study, the investigators compared their proposed MRI measure with standard examinations to show that MRI can provide additional information for therapeutic evaluation [8]. Nonetheless, limited scientific evidence was provided by these studies, which were case series with small numbers of patients.

The three studies included in this review investigated the use of MRI to quantify therapeutic response to MLD, a common component of the complex decongestive therapy that mobilizes lymph stasis from superficial tissue to deep lymphatic circulation [13]. The effectiveness of MLD remains controversial because detection of changes in lymphedema tissue is difficult with conventional examinations, such as volumetric measurement [8]. In the study by Donahue et al. [8], deep-tissue $T_{2}$ (an MRI measure) increased after MLD, but non-MRI measures did not capture significant changes.

We acknowledge the limitations of this systematic review, such as potential bias in the collection and analysis of information presented in each study. Moreover, we searched only the PubMed/ MEDLINE and Scopus databases. We also acknowledge that the studies included in this review measured the characteristics of healthy volunteers, which was not described in this study because our analysis was focused on their patients who underwent therapy $[8,12,13]$. However, this systematic review provides information on the use of MRI to evaluate therapy for BCRL, which is an important topic because of both lymphedema prevalence and debate about the efficacy of lymphedema therapies [14]. We encourage further studies that investigate the cost-effectiveness of examinations in this setting.

In summary, use of MRI for therapy evaluation in BCRL was investigated in three case series of patients undergoing MLD. These studies showed that three quantitative measurements were feasible: lymphatic vessel cross-sectional area, $\mathrm{T}_{2}$, and CEST. In one study, MRI detected post-therapeutic changes that were not detected with standard examinations. More studies with greater numbers of patients that compare proposed MRI measures with those of standard examinations are necessary to shed light on the topic.
NOTES

\section{Conflict of interest}

No potential conflict of interest relevant to this article was reported.

\section{Authors contribution}

Conceptualization: AJ Forte, D Boczar, S Kassis, MT Huayllani, SA McLaughlin. Data curation: AJ Forte, D Boczar, MT Huayllani, SA McLaughlin. Methodology: AJ Forte, D Boczar, MT Huayllani, SA McLaughlin. Project administration: AJ Forte, SA McLaughlin. Visualization: AJ Forte, S Kassis, SA McLaughlin. Writing - original draft: D Boczar, MT Huayllani. Writing - review \& editing: AJ Forte, D Boczar, S Kassis, MT Huayllani, SA McLaughlin.

\section{ORCID}

Antonio Jorge Forte https://orcid.org/0000-0003-2004-7538 Maria T. Huayllani https://orcid.org/0000-0003-2690-1635

\section{REFERENCES}

1. Newman B, Lose F, Kedda MA, et al. Possible genetic predisposition to lymphedema after breast cancer. Lymphat Res Biol 2012;10:2-13.

2. Gallagher K, Marulanda K, Gray S. Surgical intervention for lymphedema. Surg Oncol Clin N Am 2018;27:195-215.

3. Weiss M, Baumeister RG, Frick A, et al. Lymphedema of the upper limb: evaluation of the functional outcome by dynamic imaging of lymph kinetics after autologous lymph vessel transplantation. Clin Nucl Med 2015;40:e117-23.

4. Cormier JN, Askew RL, Mungovan KS, et al. Lymphedema beyond breast cancer: a systematic review and meta-analysis of cancer-related secondary lymphedema. Cancer 2010; 116:5138-49.

5. DiSipio T, Rye S, Newman B, et al. Incidence of unilateral arm lymphoedema after breast cancer: a systematic review and meta-analysis. Lancet Oncol 2013;14:500-15.

6. Ozcinar B, Guler SA, Kocaman N, et al. Breast cancer related lymphedema in patients with different loco-regional treatments. Breast 2012;21:361-5.

7. McLaughlin SA, Wright MJ, Morris KT, et al. Prevalence of lymphedema in women with breast cancer 5 years after sentinel lymph node biopsy or axillary dissection: objective measurements. J Clin Oncol 2008;26:5213-9.

8. Donahue PM, Crescenzi R, Scott AO, et al. Bilateral changes in deep tissue environment after manual lymphatic drainage in patients with breast cancer treatment-related lymphede- 
ma. Lymphat Res Biol 2017; 15:45-56.

9. Kim L, Jeon JY, Sung IY, et al. Prediction of treatment outcome with bioimpedance measurements in breast cancer related lymphedema patients. Ann Rehabil Med 2011;35:68793.

10. Szuba A, Strauss W, Sirsikar SP, et al. Quantitative radionuclide lymphoscintigraphy predicts outcome of manual lymphatic therapy in breast cancer-related lymphedema of the upper extremity. Nucl Med Commun 2002;23:1171-5.

11. Armer JM. The problem of post-breast cancer lymphedema: impact and measurement issues. Cancer Invest 2005;23:76-
83.

12. Crescenzi R, Donahue PMC, Hartley KG, et al. Lymphedema evaluation using noninvasive 3T MR lymphangiography. J Magn Reson Imaging 2017;46:1349-60.

13. Crescenzi R, Donahue PMC, Mahany H, et al. CEST MRI quantification procedures for breast cancer treatment-related lymphedema therapy evaluation. Magn Reson Med 2020; 83:1760-73.

14. Dayan JH, Ly CL, Kataru RP, et al. Lymphedema: pathogenesis and novel therapies. Annu Rev Med 2018;69:263-76. 